\title{
THE PARAMETER DEPENDENT STURM-LIOUVILLE EIGENPROBLEM WITH AN INTERIOR SIMPLE OR DOUBLE POLE
}

\author{
THOMAS M. ACHO ${ }^{1}$ and DOMINIC P. CLEMENCE ${ }^{2}$
}

(Received 10 May, 1999; revised 5 August, 1999)

\begin{abstract}
Boundary value problems where resonance phenomena are studied are most often transformable to parameter dependent Sturm-Liouville (SL) eigenproblems with interior singularities. The parameter dependent Sturm-Liouville eigenproblem with interior poles is examined. Asymptotic approximations to the solutions are obtained using an extended Langer's method to take care of the resulting complex eigenvalues and eigenfunctions.
\end{abstract}

\section{Introduction}

We consider the self-adjoint Sturm-Liouville (SL) eigenproblem with a parameter:

$$
\begin{aligned}
& \frac{d^{2} u}{d x^{2}}+\lambda^{2}[r(x) E-q(x)] u=0, \quad I=[a, b], \quad a<0<b, \\
& u(a)=u(b)=0,
\end{aligned}
$$

where $q(x)$ has a simple or double pole in the interior of $I, \lambda$ is a real parameter and $E$ is the eigenvalue parameter. Standard SL theory permits singularities only at the end points, and for $r(x)$ and $q(x)$ real and continuous on $[a, b]$. Real eigenvalues and eigenfunctions exist even if either $r(x)$ or $q(x)$ has a pole at $a$ or $b$; and in this case Langer's asymptotic method has been shown $[3,4,8]$ to provide a uniformly approximate solution. However, such an asymptotic solution has not been obtained for (1)-(2) with interior poles. For the parameterless counterpart of (1), the existence of complex eigenvalues and eigenfunctions has been proved in [6] for the simple pole

\footnotetext{
${ }^{1}$ Department of Mathematics, Vista University, PO Box 380, Bloemfontein 9300, Republic of South Africa; e-mail: acho-tm@blenny.vista.ac.za.

${ }^{2}$ Department of Mathematics, North Carolina Agricultural and Technical State University, Greensboro, NC 27411, USA; e-mail: clemence@ncat.edu.

(C) Australian Mathematical Society 2002, Serial-fee code 1446-1811/02
} 
case and in [2] for the double pole case. Asymptotic solutions have been obtained using a direct sum approach [7], and by employing Titchmarsh-Weyl $m$-function theory [2]; for the parameterless problem. It is the purpose of the present article to provide an extension of Langer's method to the simple or double pole case with a parameter, that is, (1)-(2).

The importance of studying our system cannot be overemphasized as evidenced by an ever increasing occurrence of problems with interior singularities in fluid wave theory $([2,6,7,10]$ for example) and mathematical physics [9], where resonance phenomena are studied using WKB analysis to obtain asymptotic solutions over split intervals. We note that the equation studied in [9] may be transformed into the parameter dependent interior double pole SL problem, whence our method is also applicable to the study of Schrödinger resonances. To exploit available literature, we shall assume the specific form for our BVP is of the type

$$
\begin{aligned}
& \frac{d^{2} u}{d x^{2}}+\lambda^{2}\left[\frac{f(x)}{x^{n}}-E\right] u=0, \quad I=[a, b], \quad n=1,2, \\
& u(a)=u(b)=0, \quad a<0<b .
\end{aligned}
$$

We may re-write (3) as

$$
\frac{d^{2}}{d x^{2}}+\lambda^{2} F(x, \lambda) u=0, \quad I=[a, b],
$$

where $F(x, \lambda)=\left[f(x) / x^{n}-E\right]$.

Then from [5] or [8] we obtain the expansion of the solution for (3) in the form

$$
u=B(x, \lambda) w(\xi, \gamma)+\lambda^{-(\mu+v) /(\mu+\nu+2)} C(x, \lambda) w^{\prime}(\xi, \gamma),
$$

where $\mu$ is the sum of the orders of zeros and $v$ is the sum of the orders of poles, $w(\xi, \gamma)$ is the solution to the corresponding comparison equation, and the functions $B$ and $C$ are to be determined. Following [3] and [8], we further assume that $F, B, \xi$ and $\gamma_{k}$ have the large $\lambda$ asymptotic expansions

$$
F(x, \lambda) \sim r(x) \sum_{p=0}^{\infty} \lambda^{-p} F_{p}(x)
$$

where $F_{0}=-E, F_{p}=f(x) / x^{p}, p>0$,

$$
B(x, \lambda) \sim \sum_{p=0}^{\infty} \lambda^{-p} B_{p}(x), \quad C(x, \lambda) \sim \sum_{p=0} \lambda^{-p} C_{p}(x)
$$


and

$$
\xi(x, \lambda)=\lambda^{2 /(\mu+v+2)} \phi(x), \quad \gamma_{k}=\lambda^{(\mu+v+2) / 3} \sum_{p=0}^{\infty} \lambda^{-p} \gamma_{k p} .
$$

Thus we require $\gamma_{k p}$ for $k \leq 1$ in (8). From $[3,4]$ we have

$$
\gamma_{00}=\phi^{\prime}\left(b_{0}\right), \quad \gamma_{0 p}=\left[\phi\left(b_{0}\right)\right]^{-1}\left\{F_{p}\left(b_{0}\right)+r_{p}\left(b_{0}\right)\right\}, \quad \gamma_{1 p}=0, \quad p=1,2, \ldots,
$$

where $F(x, \lambda)$ has a pole of order $n_{j}$ at $x=b_{j}, j=0, \ldots, N$, and $r(x)=\prod_{j=0}^{N}(x-b)^{n_{j}}$.

The main object of the paper then becomes the determination of the function $\phi(x)$. This paper is organized as follows. In the next section we obtain the form of the solution in terms of parameters $\theta_{m}$ and $E_{m}$ for the simple pole case. The corresponding solution for the double pole case is obtained in Section 3. In Section 4 we present a determination of the parameters $\theta_{m}$ for both cases while Section 5 presents the computation of the eigenvalues $E_{m}$. We finally present, in Section 6, the leading order asymptotic solutions for the simple and double pole cases.

\section{The simple pole case}

The comparison equation for (3) when $n=1$ is

$$
\begin{array}{ll}
\frac{d^{2} w}{d \xi^{2}}+\frac{\gamma_{0}+\xi \gamma_{1}}{\xi} w=0, & I=[a, b] \\
w\left(a, \gamma_{1}\right)=w\left(b, \gamma_{1}\right)=0, & a<0<b .
\end{array}
$$

The eigenproblem (9) with (10), using analysis similar to that in [6], has large $\lambda$ solutions

$$
\begin{aligned}
w\left(\xi, \gamma_{1}\right) \sim & \frac{\left(2 \gamma_{0}\right)^{1 / 4}}{\gamma_{1}^{1 / 3} \sqrt{\pi}}\left(\frac{\gamma_{0}+\gamma_{1} \xi}{\xi}\right)^{-1 / 4} \\
& \times \cos \left[2 k \sin \left(\frac{\gamma_{1} \xi}{\gamma_{0}}\right)^{1 / 2}+\left(\frac{\gamma_{1} \xi}{\gamma_{0}}\right)^{1 / 2}\left(1+\frac{\gamma_{1} \xi}{\gamma_{0}}\right)^{1 / 2}-k \pi+\frac{\pi}{4}\right]
\end{aligned}
$$

and the eigenrelation would be

$$
2 k\left[\arcsin \left(\frac{-\gamma_{1} b}{\gamma_{0}}\right)^{1 / 2}+\left(\frac{-\gamma_{1} b}{\gamma_{0}}\right)^{1 / 2}\left(1+\frac{\gamma_{1} b}{\gamma_{0}}\right)^{1 / 2}\right]-k \pi+\frac{\pi}{4}=\left(n-\frac{1}{2}\right) \pi,
$$


where $k=-\gamma_{0} /\left(2 \gamma_{1}^{1 / 2}\right)$. The asymptotic solution (5) also becomes

$$
u=B(x, \lambda) w\left(\xi, \gamma_{1}\right)+\lambda^{-1 / 2} C(x, \lambda) w^{\prime}\left(\xi, \gamma_{1}\right) .
$$

On substitution of (13) into (3), employing (9) and subsequently (6)-(8), we have on equating powers of $\lambda^{-p}$ for $+p \leq-2$ that

$$
\left[\phi^{\prime}\right]^{2} \frac{\gamma_{1}[\phi(x)-\phi(\alpha)]}{\phi(x)}=\frac{f(x)-E x}{x},
$$

where $x=\alpha$ is the turning point of (3), that is, the zero of $f(x)-E x$ for the simple pole case. Thus

$$
\begin{aligned}
& \int_{\phi(-x)}^{\phi\left(0^{-}\right)} \frac{\left(s-\alpha^{*}\right)^{1 / 2}}{s} d s+\int_{\phi\left(0^{+}\right)}^{\phi(x)} \frac{s-\alpha^{*}}{s^{1 / 2}} d s \\
&=\gamma_{1}^{-1 / 2} \int_{-x}^{0^{-}}\left[\frac{f(s)-E s}{s}\right]^{1 / 2} d s+\gamma_{1}^{-1 / 2} \int_{0^{+}}^{x}\left[\frac{f(s)-E s}{s}\right]^{1 / 2} d s,
\end{aligned}
$$

where $\alpha^{*}=\phi(\alpha)$ and similarly hereafter. The split interval of integration in (15) conforms with the Direct Sum Method [7], if $\alpha^{*}$ and $\gamma_{1}$ are real so that the eigenvalues $E_{m}$ of (3) are real. An application of Langer's method in this case is straightforward. If however, $\alpha^{*}, \gamma_{1}$ and consequently $E$ are complex as would be the case when the whole interval $l$ is considered, then a suitable transformation is needed so that $\phi(x)$ still retains its properties as in [3-5] and [8]. We may re-write (3) for $n=1$ as

$$
\begin{aligned}
\frac{d^{2} u}{d x^{2}}+\lambda^{2}\left[\frac{f(x)-E x}{x}\right] u=0, & I=[a, b] \\
u(a)=u(b)=0, & a<0<b .
\end{aligned}
$$

Now suppose the turning point $\tau$ (and subsequently the complex eigenvalue $E_{m}$ ) of (3) lies at the indicated position on the complex $x$-plane, see Figure 1.

In order to apply Langer's method a suitable path of integration would be the $s$-axis, obtained by rotating the real $x$-axis through $\theta_{m}$. The transformation employed would then be

$$
x=\left(\cos \theta_{m}\right) s, \quad \theta_{m}=\tan ^{-1}\left[\frac{\operatorname{Im} E_{m}}{\operatorname{Re} E_{m}}\right],
$$

where Im denotes the imaginary part and Re denotes the real part of the eigenvalue. Thus (16) transforms to

$$
\begin{gathered}
\frac{d^{2} u_{m}}{d s^{2}}+\lambda^{2}\left[\frac{f\left(s \cos \theta_{m}\right)-\left|E_{m}\right|\left(\cos \theta_{m}\right) s}{s}\right]\left(\cos \theta_{m}\right) u_{m}=0 \\
I=\left[a \sec \theta_{m}, b \sec \theta_{m}\right],
\end{gathered}
$$




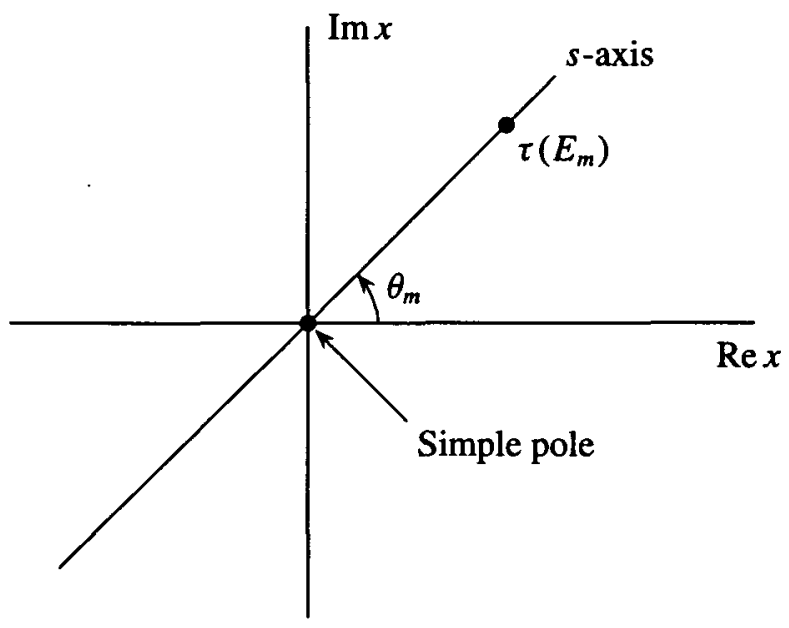

FIGURE 1.

provided $E_{m}$ is not pure imaginary. The zero of $f\left(s \cos \theta_{m}\right)-\left|E_{m}\right|\left(\cos \theta_{m}\right) s$ is real on the $s$-axis, so that $\phi(x)$ may be obtained from

$$
\left[\phi^{\prime}(s)\right]^{2} \gamma_{1} \frac{\phi(s)-\phi\left(\alpha \sec \theta_{m}\right)}{\phi(s)}=\frac{f(s \cos \theta)-\left(s \cos \theta_{m}\right)\left|E_{m}\right|}{s} \cos \theta_{m},
$$

where $s$ is as defined in (17). That is,

$$
\begin{aligned}
\int_{\phi(-s)}^{\phi\left(0^{-}\right)} & \frac{(t-\tilde{\alpha})^{1 / 2}}{t^{1 / 2}} d t+\int_{\phi\left(0^{+}\right)}^{\phi(s)} \frac{(t-\tilde{\alpha})^{1 / 2}}{t^{1 / 2}} d t \\
= & \int_{-s}^{s} \frac{\left[f\left(t \cos \theta_{m}\right)-\left|E_{m}\right|\left(t \cos \theta_{m}\right)\right]^{1 / 2}}{t^{1 / 2}} \cos ^{1 / 2} \theta_{m} d t,
\end{aligned}
$$

where $\phi\left(x \sec \theta_{m}\right)=\tilde{x}$ and similarly hereafter, and $\int_{-s}^{s} G d t=\int_{-s}^{0^{-}} G d t+\int_{0^{+}}^{s} G d t$. Evaluating the integral on the right-hand side of (20) we then have

$$
\begin{gathered}
{[\phi(-s)(\phi(-s)-\tilde{\alpha})]^{1 / 2}-[\phi(s)(\phi(s)-\tilde{\alpha})]^{1 / 2}+\tilde{\alpha} \log \left|\frac{[\phi(-s)]^{1 / 2}+(\phi(-s)-\alpha)^{1 / 2}}{\phi(s)^{1 / 2}+(\phi(s)-\alpha)^{1 / 2}}\right|} \\
=\gamma_{1}^{1 / 2} \int_{-s}^{s}\left[\frac{f\left(t \cos \theta_{m}\right)-\left|E_{m}\right|\left(t \cos \theta_{m}\right)}{t}\right]^{1 / 2} \cos ^{1 / 2} \theta_{m} d t .
\end{gathered}
$$

With $\phi(s)$ as obtained in (21) and the functions $B_{p}$ and $C_{p}$ determined as in [3-5] and [8], a substitution into (13) then gives the required solution to the BVP (3) with (4) for $n=1$. 


\section{The double pole case}

When $n=2$ in (3) the asymptotic expansion of the solution of this eigenproblem becomes

$$
u=B(x, \lambda) w\left(\xi, \gamma_{2}\right)+\lambda^{-1 / 3} C(x, \lambda) w^{\prime}\left(\xi, \gamma_{2}\right)
$$

where $w\left(\xi, \gamma_{2}\right)$ is the solution to the comparison equation

$$
\frac{d^{2} w}{d \xi^{2}}+\left(\frac{\gamma_{0}+\gamma_{1} \xi+\gamma_{2} \xi^{2}}{\xi^{2}}\right) w=0, \quad[a, b]
$$

with boundary conditions

$$
w\left(a, \gamma_{2}\right)=w\left(b, \gamma_{2}\right)=0, \quad a<0<b
$$

The solution $w\left(\xi, \gamma_{2}\right)$ is as obtained in [2]. The split interval consideration enables us to apply Langer's method as employed in the first part of Section 2, above. That is, $\phi(x)$ may be obtained from

$$
\left(\phi^{\prime}\right)^{2} \gamma_{2} \frac{\left[\phi(x)-\phi\left(\tau_{1}\right)\right]\left[\phi(x)-\phi\left(\tau_{2}\right)\right]}{(\phi(x))^{2}}=\frac{f(x)-E x^{2}}{x^{2}}
$$

so that

$$
\int_{\phi(-x)}^{\phi(x)} \frac{\left[\left(t-\tau_{1}^{*}\right)\left(t-\tau_{2}^{*}\right)\right]^{1 / 2}}{t} d t=\gamma_{2}^{-1 / 2} \int_{-x}^{x} \frac{\left[f(t)-E t^{2}\right]^{1 / 2}}{t} d t
$$

from which $\phi(x)$ may be obtained by a direct application of Langer's method, provided the eigenvalues and turning points are real. However, if a second solution other than the 'distinguished' solution (see [7]) is considered (which is normally the case when the whole interval is relevant), the eigenvalues, the turning points and $\phi(x)$ are all complex. In this context we need to use a transformation similar to (17). The presence of two turning points which may be separated or may lie on the same half plane (as in Tables 1 and 2 of [2]) gives rise to two transformations.

The turning points lie in the first and third quadrants (refer to Figure 2) but a similar transformation would be obtained if they lie on any part of the complex $x$-plane.

A rotation of the positive real $x$-axis through $\theta_{m}^{+}$gives the transformation

$$
x=\left(\cos \theta_{m}^{+}\right) s, \quad \theta_{m}^{+}=\tan ^{-1}\left[\frac{\operatorname{Im} E_{m}^{+}}{\operatorname{Re} E_{m}^{+}}\right],
$$

and similarly the negative real $x$-axis through $\theta_{m}^{-}$gives the transformation

$$
-x=\left(\cos \theta_{m}^{-}\right) s, \quad \theta_{m}^{-}=\tan ^{-1}\left[\frac{\operatorname{Im} E_{m}^{-}}{\operatorname{Re} E_{m}^{-}}\right] .
$$




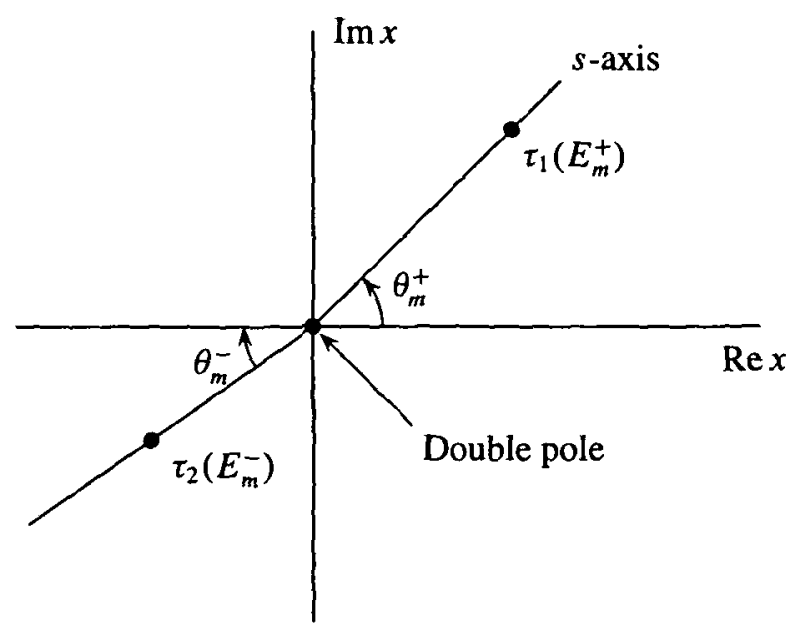

FIGURE 2.

These then transform (3) to

$$
\begin{gathered}
\frac{d^{2} u_{m}}{d x^{2}}+\lambda^{2}\left[\frac{f\left(s \cos \theta_{m}^{ \pm}\right)-\left|E_{m}\right|\left(s \cos \theta_{m}^{ \pm}\right)}{s^{2}}\right] u_{m}=0 \\
I=\left[a \sec \theta_{m}^{-}, b \sec \theta_{m}^{+}\right],
\end{gathered}
$$

where either only the upper signs or only the lower signs are chosen. The differential equation from which we may compute $\phi(x)$ will then be

$$
\begin{gathered}
\gamma_{2} \frac{\left[\phi^{\prime}( \pm s)\right]^{2}}{[\phi( \pm s)]^{2}}\left[\phi(s)-\phi\left(\tau_{1} \sec \theta_{m}^{ \pm}\right)\right]\left[\phi(s)-\phi\left(\tau_{2} \sec \theta_{m}^{ \pm}\right)\right] \\
=\frac{\left[f\left( \pm s \cos \theta_{m}^{ \pm}\right)-\left|E_{m}\right|\left( \pm s \cos \theta_{m}^{ \pm}\right)\right]}{s^{2}} .
\end{gathered}
$$

That is,

$$
\begin{aligned}
\int_{\phi(-s)}^{\phi\left(0^{-}\right)} & \frac{\left[\left(t-\tilde{\tau}_{1}\right)\left(t-\tilde{\tau}_{2}\right)\right]^{1 / 2}}{t} d t+\int_{\phi\left(0^{+}\right)}^{\phi(s)} \frac{\left[\left(t-\tilde{\tau}_{1}\right)\left(t-\tilde{\tau}_{2}\right)\right]^{1 / 2}}{t} d t \\
= & \gamma_{2}^{-1 / 2} \int_{-s}^{0^{-}} \frac{\left[f\left(-t \cos \theta_{m}^{-}\right)-\left|E_{m}\right|\left(t \cos \theta_{m}^{-}\right)^{2}\right]^{1 / 2}}{t} d t \\
& +\gamma_{2}^{-1 / 2} \int_{0^{+}}^{s} \frac{\left[f\left(t \cos \theta_{m}^{+}\right)+\left|E_{m}\right|\left(t \cos \theta_{m}^{+}\right)^{2}\right]^{1 / 2}}{t} d t .
\end{aligned}
$$

The determination of $B_{p}$ and $C_{p}$ as in [3,4] and [8], with $\phi(s)$ as obtained in (28) completes the asymptotic approximation to the solution of $(3)$ for the double pole case as presented in (22). 


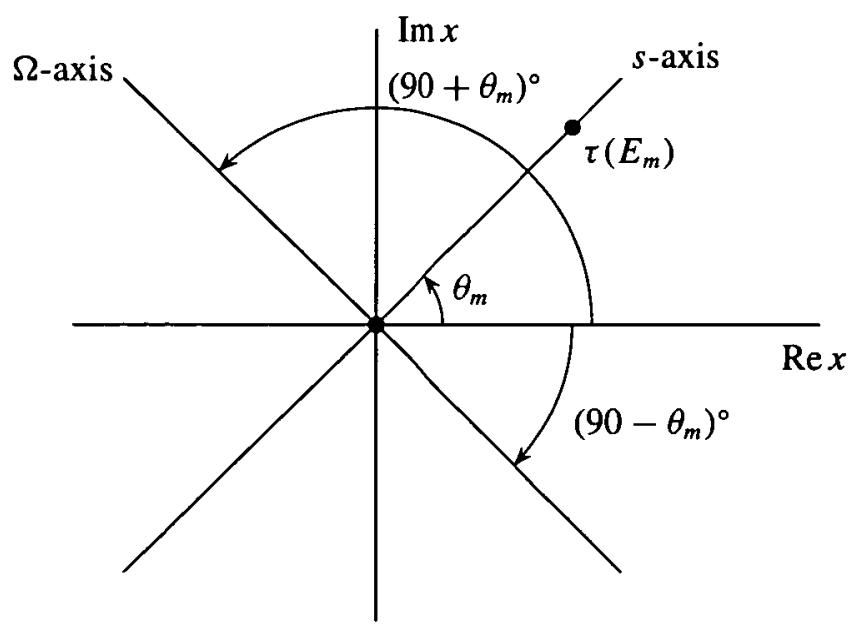

FIGURE 3 .

\section{Determination of $\theta_{m}$}

At this point we need an additional equation in each case for the determination of $\theta_{m}$, since the equations (21) and (28) for the computation of $\phi(x)$ contain unknowns $E_{m}$ and $\theta_{m}$. The unknown $E_{m}$ will be determined in the next section, once $\theta_{m}$ is computed. We shall tackle this problem by constructing an $\Omega$-axis perpendicular to the $s$-axis. Along this axis, there are no turning points since $\tau_{j}$ lies on the $s$-axis (see Figure 3).

Under this transformation, namely $x=\left[ \pm \sin \theta_{m}\right] \Omega$ and $\theta_{m}=\arg \left(E_{m}\right)$, the BVP (3) with (4) devoid of $E_{m}$ is

$$
\begin{gathered}
\frac{d^{2} u_{m}}{d \Omega^{2}}+\lambda^{2}\left[\frac{f\left( \pm \Omega \sin \theta_{m}\right)}{\left( \pm \Omega \sin \theta_{m}\right)^{n}}\right]\left( \pm \sin \theta_{m}\right)^{2} u_{m}=0, \quad I=\left[-a \csc \theta_{m}, b \csc \theta_{m}\right] \\
u_{m}\left(-a \csc \theta_{m}\right)=u_{m}\left(b \csc \theta_{m}\right)=0 .
\end{gathered}
$$

4.1. Determining $\theta_{m}$ for the simple pole eigenproblem Now for $n=1$, that is, the simple pole case, (29) becomes

$$
\frac{d^{2} u_{m}}{d \Omega^{2}}+\lambda^{2}\left[\frac{f\left( \pm \Omega \sin \theta_{m}\right)}{\Omega}\right]\left( \pm \sin \theta_{m}\right) u_{m}=0
$$

The comparison equation to $(31)$ is

$$
w_{m}^{\prime \prime}+\gamma_{0} \xi^{-1} w_{m}=0
$$


The solutions are cylindrical functions of order 1 , that is,

$$
w_{m}(\xi)=\xi^{1 / 2}\left[\alpha_{m} J_{1}\left(2 \gamma_{0}^{1 / 2} \xi^{1 / 2}\right)+\beta_{m} Y_{1}\left(2 \gamma_{0}^{1 / 2} \xi^{1 / 2}\right)\right]
$$

where $\alpha_{m}, \beta_{m}$ are constants, and $\xi$ is defined as $\xi=\lambda^{2 / 3} \phi(\Omega)$ and

$$
\gamma_{0}=\lambda^{4 / 3} \sum_{p=0}^{\infty} \lambda^{-p} \gamma_{0 p}
$$

with $\gamma_{00}$ arbitrary. The absence of turning points in (32) enables us to invoke Theorem 3 of [7] without the 'full interval' analysis of the earlier sections. The distinguished solution of this case would be the Bessel function $J_{1}$, so that

$$
w_{m}(\Omega)= \begin{cases}\lambda^{1 / 3} \phi_{m}(\Omega) \alpha_{m} J_{1}\left(2 \gamma_{0}^{1 / 2} \lambda^{1 / 3} \phi_{m}\right), & 2\left(\gamma_{0} \lambda\right)^{1 / 2} \phi_{m} \in\left[0, b \csc \theta_{m}\right] \\ 0, & 2\left(\gamma_{0} \lambda\right)^{1 / 2} \phi_{m} \in\left[-a \csc \theta_{m}, 0\right]\end{cases}
$$

In order to satisfy the boundary conditions on $\left[0, b \csc \theta_{m}\right]$, we require that along the $\Omega$-axis $J_{1}\left[2 \gamma_{0}^{1 / 2} \lambda^{1 / 2} \phi_{m}\left(b \csc \theta_{m}\right)\right]=0$.

Since $\phi_{m}$ and $\gamma_{0}$ are real, the $m^{t h}$ positive zero of $J_{1}, Z_{m}$, may be computed $([1,11])$ as follows:

$$
2 \gamma_{0}^{1 / 2} \lambda^{1 / 2} \phi_{m}\left(b \csc \theta_{m}\right)=Z_{m}
$$

so that

$$
\theta_{m}=\csc ^{-1}\left[\frac{1}{b} \phi_{m}^{*}\left(\frac{Z_{m}}{2} \gamma_{0}^{1 / 2} \lambda^{1 / 2}\right)\right]
$$

where $\phi_{m}^{*}$ is the inverse function of $\phi_{m}$. Thus $\theta_{m}$ would be finally obtained if $\phi_{m}^{*}$ appearing in (33) is computed.

We now apply Langer's method to the transformed BVP (29) with (30) along the $\Omega$-axis. Namely, $\phi(x)$ may then be obtained from the differential equation

$$
\left(\phi^{\prime}\right)^{2} \frac{\gamma_{0}}{\phi}=\frac{f\left( \pm \Omega \sin \theta_{m}\right)}{\Omega}\left( \pm \sin \theta_{m}\right), \quad \phi=\phi(\Omega),
$$

that is,

$$
\int_{\phi(0)}^{\phi(\Omega)} \frac{d t}{t}=\frac{1}{\gamma_{0}^{1 / 2}}\left[\int_{0}^{\Omega}\left(\frac{f\left(t \sin \theta_{m}\right)}{t} \sin \theta_{m}\right)^{1 / 2} d t\right] .
$$

For a specific $f(x),(33)$ and (34) may be solved simultaneously for $\phi_{m}$ and $\theta_{m}$. This completes the determination of $\theta_{m}$ for the simple pole case. 
4.2. Determining $\theta_{m}$ for the double pole eigenproblem When $n=2$, the transformed BVP (29) becomes

$$
\begin{gathered}
\frac{d^{2} u_{m}}{d \Omega^{2}}+\lambda^{2}\left[\frac{f\left( \pm \Omega \sin \theta_{m}\right)}{\Omega^{2}}\right] u_{m}=0, \\
u_{m}\left(-a \csc \theta_{m}\right)=u_{m}\left(b \csc \theta_{m}\right)=0 .
\end{gathered}
$$

The comparison equation to (35) is

$$
w_{m}^{\prime \prime}+\gamma_{0} \xi^{-2} w_{m}=0
$$

which has a solution $w_{m}(\xi)=\alpha_{m} \xi^{1 / 2\left(1+\sqrt{1-4 \gamma_{0}}\right)}+\beta_{m} \xi^{1 / 2\left(1-\sqrt{1-4 \gamma_{0}}\right)}$, where $\alpha_{m}, \beta_{m}$ are constants. In this case

$$
\xi=\lambda^{1 / 2} \phi(\Omega), \quad \gamma_{0} \sim \lambda^{2} \sum_{p=0}^{\infty} \lambda^{-p} \gamma_{0 p}
$$

As usual we appeal to Theorem 3 of [7] and choose the distinguished solution as $w_{m}(\xi)=\alpha_{m} \xi^{1 / 2\left(1+\sqrt{1-4 \gamma_{0}}\right)}$ so that, on using (36),

$$
w_{m}(\Omega)= \begin{cases}\alpha_{m}\left[\lambda^{1 / 2} \phi(\Omega)\right]^{1 / 2\left(1+\sqrt{\left.1-\alpha_{\gamma 0}\right)}\right.} & \text { on } I=\left[0, b \csc \theta_{m}\right], \\ 0 & \text { on } I=\left[-a \csc \theta_{m}, 0\right]\end{cases}
$$

On application of the boundary condition at $b$ we get

$$
\theta_{m}=\csc ^{-1}\left[\frac{\phi^{*}(o)}{b}\right]
$$

Going by Langer's method, along the $\Omega$-axis, $\phi(\Omega)$ may be obtained from

$$
\left(\phi^{\prime}(\Omega)\right)^{2} \frac{\left(\gamma_{0}+\gamma_{1} \phi(\Omega)\right)}{(\phi(\Omega))^{2}}=\frac{f\left( \pm \Omega \sin \theta_{m}\right)}{\Omega^{2}}
$$

so that

$$
\int_{\phi(0)}^{\phi_{m}(\Omega)} \frac{\left(\gamma_{0}+\gamma_{1} t\right)^{1 / 2}}{t} d t=\int_{0}^{\Omega} \frac{\left[f\left(t \sin \theta_{m}\right)\right]^{1 / 2}}{t} d t
$$

The integration on the left-hand side would be done in the limit just as it should be on the right-hand side. Thus we obtain $\theta_{m}$ and $\phi_{m}$ from (37) and (38). This completes the determination of $\theta_{m}$ for the double pole case. 


\section{Eigenvalues $E_{n}$}

We recall (18):

$$
\frac{d^{2} u_{m}}{d s^{2}}+\lambda^{2}\left[\frac{f\left(s \cos \theta_{m}\right)-s\left|E_{m}\right| \cos \theta_{m}}{s}\right] \cos \theta_{m} u_{m}=0
$$

Comparing this with (1.1) of [4] we have

$$
\begin{aligned}
u(z, \lambda) & \rightarrow u_{m}(s, \lambda)=\frac{-f\left(s \cos \theta_{m}\right)}{s} \cos \theta_{m}, \\
E & \rightarrow-\left|E_{m}\right|\left(\cos ^{2} \theta_{m}\right) .
\end{aligned}
$$

And going by [8] we have

$$
F_{o}(z) \rightarrow\left[\frac{f\left(s \cos \theta_{m}\right) \cos \theta_{m}}{s}-\left|E_{m}\right| \cos ^{2} \theta_{m}\right] .
$$

Thus with analysis similar to that in [5]; if $\gamma_{0}$ is an eigenvalue of (9) then for the $n$-th eigenvalue

$$
\gamma_{1}=g_{m}\left(\gamma_{0}, \gamma_{2}, \ldots, \gamma_{n}\right)
$$

Using the expansion of $\gamma_{k}(\lambda),(40)$ becomes

$$
\lambda \sum_{t=0}^{\infty} \gamma_{0 t}\left|E_{m}\right| \lambda^{-t}=g_{m}\left[\lambda \sum \gamma_{1 t}\left|E_{m}\right| \lambda^{-t}\right] .
$$

The solution for $\left|E_{m}\right|$ in (39) gives the $m$-th eigenvalue $\left|E_{m}(\lambda)\right|$ for (18). With $\gamma_{0}$ as obtained in [8], and $\tau_{1}$ being the turning point in the right half plane say (that is, the zero of $\left.f\left(s \cos \theta_{m}\right)-s\left(\left|E_{m}\right| \cos ^{2} \theta_{m}\right)\right)$ then from [4] we may write

$$
\frac{\lambda}{\pi} \int_{0}^{\pi_{1}\left(\left|E_{m}\right|\right)}\left[\frac{f\left(s \cos \theta_{m}\right)}{s} \cos \theta_{m}-\left|E_{m}\right| \cos ^{2} \theta_{m}\right] d s+O\left(\lambda^{-1}\right)=g_{m} .
$$

If we set $\left|E_{m}\right|=\left|E_{m}^{0}(\lambda)\right|+\lambda^{-1}\left|E_{m}^{1}(\lambda)\right|+O\left(\lambda^{-1}\right)$ as in [5] and expand the left-hand side of (40) in powers of $\lambda^{-1}$ and equate coefficients of like powers of $\lambda^{-1}$, we have

$$
\frac{\lambda}{\pi} \int_{0}^{r_{1}\left|E_{m}\right|}\left[f\left(s \cos \theta_{m}\right) \cos \theta_{m}-\left|E_{m}\right| \cos ^{2} \theta_{m}\right] d s=g_{m},
$$

where $g_{m}=O\left(\lambda^{-1}\right)$.

This gives the first two terms in the asymptotic expansions for $\left|E_{m}\right|$ from which we may then obtain $\left|E_{m}\right|$ as $E_{m}=\left|E_{m}\right| \cos \theta_{m}+i\left|E_{m}\right| \sin \theta_{m}$. This completes the determination of $E_{m}$.

The procedure for the double pole is very much the same except that (27) will be used in place of (18) and $g_{m}$ would be such that $\gamma_{2}=g_{m}\left(\gamma_{0}, \gamma_{1}, \gamma_{3}, \ldots, \gamma_{m}\right)$ in place of (40). 


\section{The asymptotic solutions}

6.1. First order pole case Employing the method for the determination of the functions $B_{p}$ and $C_{p}$ as in [4,5] and [8], we obtain $B_{0}=\left(\phi^{\prime}(s)\right)^{-1 / 2}$ and $C_{0}=0$, where $\phi(s)$ would be as in (21). From [8], $\left(\phi^{\prime}(s)\right)^{-1 / 2}=\lambda^{1 / 4}\left[\xi^{\prime}(s, \lambda)\right]^{-1 / 2}$. For (11) and (13), the asymptotic expansions of the solution to the BVP (3) with (4) for $n=1$ may then be written as

$$
\begin{aligned}
u \sim & \left(\phi^{\prime}(s)\right)^{-1 / 2}\left[\frac{\left(2 \gamma_{0}\right)^{1 / 4}}{\gamma_{1}^{1 / 3} \sqrt{\pi}}\left(\frac{\gamma_{0}+\gamma_{1} \xi}{\xi}\right)^{-1 / 4}\right. \\
& \left.\times \cos \left[2 k \sin ^{-1}\left(\frac{\gamma_{1} \xi}{\gamma_{0}}\right)+\left(\frac{-\gamma_{1} \xi}{\gamma_{0}}\right)^{1 / 2}\left(\frac{\gamma_{0}+\gamma_{1} \xi}{\gamma_{0}}\right)^{1 / 2}-k \pi+\frac{\pi}{4}\right]\right]+O\left(\lambda^{-3 / 4}\right),
\end{aligned}
$$

where $\gamma_{1}$ is as obtained in (12), and $s=\left(\sec \theta_{m}\right) x$ as in (17).

For the split interval, in which case the eigenvalues are real and only the distinguished solution is considered, with $\phi(x)$ as in (15) we find that

$$
u \sim \alpha_{m}\left(\phi^{\prime}(x)\right)^{-1 / 2} M_{k, \mu}\left(\xi\left(x, \gamma_{1}\right)\right)+O\left(\lambda^{-3 / 4}\right),
$$

where $w_{m}=\alpha_{m} M_{k, \mu}\left(\xi\left(x, \gamma_{1}^{(m)}\right)\right)$ as in [2] and $\gamma_{1}^{(m)}$ is obtained from the $m^{\text {th }}$ positive zero of the Whittaker function $M_{k, \mu}(\cdot)$. It is worth noting that in computing the integral for $\phi(x)$ from (15), for this case $\int_{-x}^{0^{-}} d s=0$ as a result of the application of Theorem 3 of [7].

6.2. Second order pole case With similar calculations we find that for the double pole case

$$
B_{0}=\left(\phi^{\prime}(s)\right)^{-1 / 2} \text { and } C_{0}=0,
$$

where $\phi(s)$ is as obtained in (28).

A substitution of the solution to the comparison equation (23) with (24), and (41) into (22) gives the asymptotic solution for the BVP (3) with (4) for $n=2$ (the double pole case). That is,

$$
u \sim\left(\phi^{\prime}(s)\right)^{-1 / 2}\left[\begin{array}{ll}
\psi_{+}\left(\xi, \gamma_{2}\right), & \xi \in[a, 0), \\
\psi_{-}\left(\xi, \gamma_{2}\right), & \xi \in[0, b],
\end{array}\right.
$$

where $\psi_{+}(\cdot)$ and $\psi_{-}(\cdot)$ are as obtained in [2] and $\xi$ takes the form $\xi=\lambda^{1 / 3} \phi(x)$. For the split interval consideration $\phi(x)$ would be obtained from (26) and the asymptotic solution will take the form

$$
u \sim \alpha_{m}\left(\phi^{\prime}(x)\right)^{-1 / 2} M_{k, \mu}\left(\xi\left(x, \gamma_{2}\right)\right)+O\left(\lambda^{-5 / 6}\right),
$$

where $M_{k, \mu}\left(\xi\left(x, \gamma_{2}\right)\right)$ is as defined in [2] and $\gamma_{2}$ here will be obtained from the zeros of the Whittaker function $M_{k, \mu}(\cdot)$. 


\section{Concluding remarks}

The split interval analysis for both cases (simple pole and double pole) is similar though the eigenrelations are different as is evident in [2]. The similarity in the analysis of the comparison equations is principally because one half of the complex plane is considered and the connection formula for both cases would be the same if the turning points in the double pole case coalesce.

It is obvious from the analysis that except for the split interval consideration, where the only difference in the asymptotic solutions would be in the variable parameter $\mu$ (of the Whittaker function for the double pole case and the resulting eigenrelations), the asymptotic solutions would be very different in both cases. An important feature of these solutions as presented in Section 6 is that the solution for the double pole case has two asymptotic representations for the two arms of the real $x$-axis, whereas the simple pole case has a single asymptotic representation. Also, for large $\lambda$, we see that the first term for the double pole case would be a better asymptotic approximation to its solution than would be the first term of the simple pole case.

\section{References}

[1] M. Abramowitz and I. Stegun, Handbook of mathematical functions (Dover, New York, 1965).

[2] T. M. Acho and D. P. Clemence, "Sturm-Liouville eigenproblems with an interior double pole", ZAMP 46 (1995) 459-474.

[3] D. U. Anyanwu, "Uniform asymptotic solutions of second order linear ordinary differential equations with singular points I. Formal theory", J. Math. Anal. Appl. 134 (1989) 329-354.

[4] D. U. Anyanwu, "Uniform asymptotic solutions of second order linear ordinary differential equations with singular points II. Some expansions", J. Math. Anal. Appl. 134 (1989) 355-378.

[5] D. U. Anyanwu and J. B. Keller, "Asymptotic solutions of eigenvalue problems for second order ordinary differential equations", Comm. Pure Appl. Math. 28 (1975) 753-763.

[6] J. P. Boyd, "Sturm-Liouville eigenproblems with an interior pole", J. Math. Phys. 22 (1981) 1575-1590.

[7] W. N. Everitt, J. Gunson and A. Zettl, "Some comments on Sturm-Liouville eigenvalue problems with interior singularities", ZAMP 38 (1987) 813-838.

[8] R. V. S. Lynn and J. B. Keller, "Uniform asymptotic solutions of second order linear ordinary differential equations with turning points", Comm. Pure Appl. Math. 23 (1970) 379-489.

[9] M. A. Shubov, "High-energy asymptotics of resonances for three-dimensional Schrödinger operator with screened Coulomb potential", J. Math. Phys. 35 (1994) 656-679.

[10] A. J. Simmons, "Planetary-scale disturbances in the polar winter stratosphere", Quart. J. R. Met. Soc. 100 (1974) 76-108.

[11] L. J. Slater, Confluent hypergeometric functions (Cambridge University Press, 1960). 